\title{
Effects of friction on stress on a plate penetrating into granular media
}

Chuan-Ping Liu (刘传平) ${ }^{1,2, *}$, Chuan-YuWu (邬传宇) ${ }^{2, \dagger}$, Chao Zheng (郑超) ${ }^{2}$, Li Wang (王立) ${ }^{l}$

${ }^{l}$ School of Energy and Environment Engineering, University of Science and Technology Beijing, 100083, China

${ }^{2}$ Department of Chemical and Process Engineering, University of Surrey, GU2 7XH, UK

*cpliu@ustb.edu.cn

c.y.wu@surrey.ac.uk

Abstract: The penetration of a plate into granular media was analyzed, and the effects of particle-plate and particleparticle frictions, penetration direction, and initial plate orientation were examined. Results showed that stress was directly proportional to immersion depth for frictionless particles but jumped at the bed surface and then increased linearly for frictional particles. Moreover, stress was mostly independent of the penetration direction when the plate was frictionless. However, initial orientation always had an effect regardless of whether the plate was frictional or frictionless. Furthermore, a theoretical model was developed for stress analysis. This model revealed that friction on the plate essentially affected stress via changing the push angle of the particles that were in contact with the plate.

Keywords: Intruder; Granular media; Friction; Resistive force

\section{Nomenclature}

$d \quad$ average diameter of the particle

$K_{\sigma} \quad$ dimensionless stress increasing number

$K_{A} \quad$ stress transition coefficient in Zone I

$K_{B} \quad$ stress transition coefficient in Zones II and III

$f_{0} \quad$ stress jump

$f_{\sigma} \quad$ normal stress on the plate

$f_{\tau} \quad$ shear stress on the plate

$f_{m g} \quad$ shear stress induced by the friction and gravity of particles

$g \quad$ gravity acceleration

$h \quad$ immersion depth of the plate

$v \quad$ penetration velocity

$\alpha \quad$ push angle of the plate

$\beta \quad$ initial orientation angle of the plate

$\gamma \quad$ penetration angle of the plate

$\rho \quad$ density of the solid particle

$\zeta$ solid volume fraction of the solid particle

$\eta \quad \angle \mathrm{AOB}$

$\xi \quad$ proportion factor related to particle-plate friction

$\theta \quad \angle \mathrm{CAD}$

$\lambda_{\sigma} \quad$ normal stress gradient per unit depth

$\mu_{s} \quad$ coefficients of static friction

$\mu_{r} \quad$ coefficients of rolling friction

$\varphi \quad$ internal friction angle of the particle

$\varphi_{p i} \quad$ friction angle of the plate

$\chi \quad \angle \mathrm{OBA}$

$\psi \quad \angle \mathrm{OAP}$

$\omega \quad$ intersection angle between stress $f$ and the normal direction of the plate

19

\section{Introduction}

Granular materials can deform and flow under stress. However, they occasionally exhibit characteristics that differ from traditional fluids ${ }^{[1,2]}$ because particles interact with their neighbors through contact forces. A representative case of a large object, namely, an intruder moving in granular material, has received increasing attention from physicists and engineers because of its ubiquitous applications in impact cratering ${ }^{[3,4]}$, ground vehicles ${ }^{[5,6]}$, soil plowing ${ }^{[7,8]}$, and excavation ${ }^{[9]}$.

When an intruder penetrates vertically into a granular bed at a low speed, it experiences an opposite drag force, called the quasi-static frictional force, which is independent of penetration velocity. In accordance with resistive force theory ${ }^{[10,11]}$, an intruder of complex geometries can be partitioned into infinitesimal segments, and the force on the intruder can be obtained from the integration of stresses on the segments as follows:

$$
\boldsymbol{F}=\int_{S} f d A_{s}
$$

where $S$ is the surface of the intruder, $d A_{s}$ is the area of the segment, and $\boldsymbol{f}$ is the stress on $A_{s}$. In previous researches 
${ }^{[12,13,14]}$, stress is usually considered to be directly proportional to the hydrostatic-like pressure from the above granular bed, i.e., $f=K \rho \zeta g h$, where $\rho$ is the density of the solid particle, $\zeta$ is the solid volume fraction, $g$ is the gravity acceleration, $h$ is the immersion depth, and $K$ is the proportional coefficient. Brzinski III ${ }^{[12]}$ proposed an expression for the coefficient $K=C \tan \varphi$, where $\varphi$ is the internal friction angle and $C=26 \pm 3$ for experiments with 300-micron glass spheres. Kang ${ }^{[13]}$ proposed a model wherein $K$ is a single-value function of $\varphi$ for the calculation of penetration force.

Consequently, stress is influenced by the physical parameters of particles; however, the friction between the intruder and particles is not included in these models. Recently, Zheng ${ }^{[15]}$ examined the impact of an intruder into a two-dimensional granular bed and showed that an intruder with a gear surface has a smaller depth, speed, and impact time than an intruder with a smooth surface. Hence, the friction between an intruder and particles is anticipated to play a role in the penetration of intruders.

Therefore, the current study aimed to explore whether and how particle-intruder and particle-particle friction affect the force on an intruder penetrating in granular media. The penetration of a plate into a granular bed from the bed surface was numerically analyzed. Our results indicated that interparticle friction resulted in a stress jump at the bed surface. Moreover, the friction between the plate and the particles caused a stress difference in oblique penetration but negligibly affected stress when the plate was penetrating vertically. Furthermore, a theoretical model for stress calculation was developed to elucidate the effect of friction ${ }^{[15]}$; this model was also helpful to clarify the mechanism of granular flow ${ }^{[16,17]}$, which was different from the traditional fluid.

\section{Computational model}

A discrete element method (DEM) was adopted to simulate the penetration of a plate into a granular bed from which the forces on the plate surface were determined. As shown in Fig. 1, the computational domain was $100 \mathrm{~mm}$ in width $(W)$ and $10 \mathrm{~mm}$ in thickness $(\delta)$. The boundaries in the horizontal and vertical directions were periodic, i.e., the particles moving out of the domain could re-enter from the opposite side with the same kinetics. Glass particles with a density $(\rho)$ of $2,546 \mathrm{~kg} / \mathrm{m}^{3}$, an average diameter $(d)$ of $1.0 \mathrm{~mm}$, and a standard deviation normal distribution of 0.05 were used to randomly generate the bed up to an initial height $(H)$ of $80 \mathrm{~mm}$. A plate with a width of $10 \mathrm{~mm}$ $(w, w=\delta)$ and a thickness of $0.2 \mathrm{~mm}$ was used as the intruder that penetrated into the bed at a constant velocity $(v)$. The length of the plate $(l)$ was changed under different simulation conditions. The initial orientation angle $(\beta)$ of the plate was defined as the angle between the plate surface and horizontal direction with the range of $-\pi / 2-\pi / 2$, and the penetration angle $(\gamma)$ between the penetration and horizontal direction had the range of $0-\pi / 2$. The motion of the particles was computed by using Newton's second law, and contact between particles and contact between particles and the plate were described by utilizing the Hertz-Mindlin model ${ }^{[18]}$. The Poisson's ratios of the particles and the plate were 0.24 and 0.4 , respectively, and their Young's moduli were $2.2 \times 10^{8}$ and $1.0 \times 10^{8} \mathrm{~Pa}$, respectively. The coefficient of restitution between the particles and the plate was set as 0.5 .

During the simulation, the plate penetrated from the bed surface at a constant velocity (v), i.e., the immersion depth $(h)$ was gradually increased. Here, the immersion depth was defined as the vertical distance from the bed surface to the center of the plate. The drag force on plate was calculated from the contact force between the particles and the plate surface. Normal stress $\left(f_{\sigma}\right)$ and shear stress $\left(f_{\tau}\right)$ were calculated as the normal and shear forces per unit area. Only the forces on the head-on surface were considered to eliminate the influence of the intruder ends. The coefficients of static and rolling friction between particles $\left(\mu_{s p p}\right.$ and $\left.\mu_{r p p}\right)$, and those between particles and the plate $\left(\mu_{s p i}\right.$ and $\left.\mu_{r p i}\right)$ were changed under different simulation conditions to discuss the effects of friction on stress.

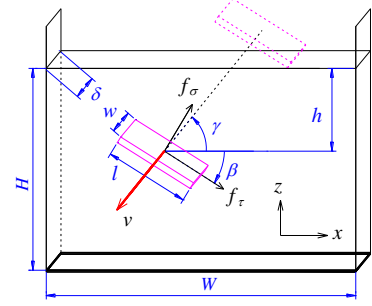

Fig. 1 Penetration of a plate into a granular bed. Simulation domain is a box bed with periodic boundaries, and the plate penetrates from the bed surface.

\section{Stresses on the plate}

Frictionless particles without any interparticle friction $\left(\mu_{s p p}=0\right.$ and $\left.\mu_{r p p}=0\right)$ were used to randomly generate the 
bed, and the bed solid volume fraction ( $\zeta$ ) was 0.646 . When a frictionless plate penetrated into this bed, only $f_{\sigma}$ existed, and $f_{\tau}=0 . f_{\sigma}$ increased and was proportional to the immersion depth. Hence, this quasi-static stress was independent of plate size and penetration velocity, which met the previous researches ${ }^{[13,19,20]}$. The normal force increased proportionally with the increase in plate length, whereas $f_{\sigma}$ remained constant (Fig. 2a). In addition, we varied the penetration velocity from $5.0 \mathrm{~mm} / \mathrm{s}$ to $20 \mathrm{~mm} / \mathrm{s}$ but exerted constant stress on the plate at the same immersion depth.

Regardless of the penetration depth, stress was always greater than the hydrostatic pressure of the granular bed $\rho \zeta g h$. Here, we defined the proportional coefficient as the dimensionless stress increasing number $K_{\sigma}=\lambda_{\sigma} / \rho \zeta g$, where $\lambda_{\sigma}$ is the normal stress gradient per unit depth corresponding to the slope of the solid lines in Fig. 2a, i.e., $\lambda_{\sigma}=\partial f_{\sigma} \partial \partial$. When $K_{\sigma}$ was large, the stress increase rate was high. The relationship between stress and immersion depth also did not change with penetration angle (Fig. 2b). Furthermore, stress was independent of penetration velocity, plate length, and penetration direction under this condition. However, stress depended on the initial orientation of the plate: it decreased when the orientation angle $(\beta)$ was increased (inset of Fig. $\mathbf{2 b}$ ).

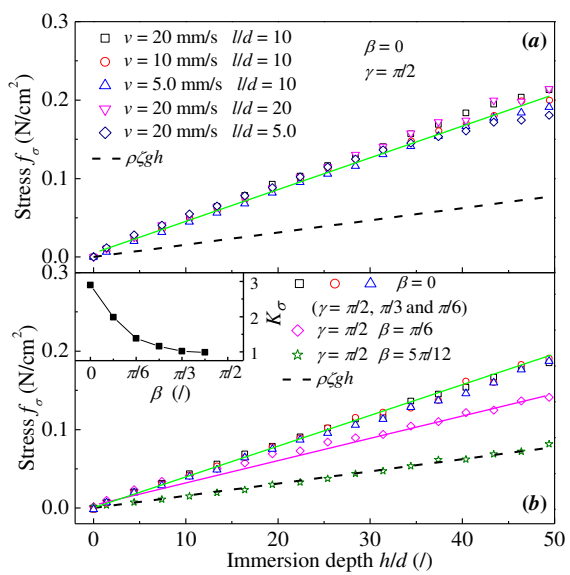

Fig. 2 Stress on the frictionless plate penetrating in the frictionless granular bed. The dotted lines represent hydrostatic pressure, and the solid lines are trend-lines. Stress is independent of plate size $l / d$, penetration velocity $v$, and penetration angle $\gamma$, and affected by its orientation angle $\beta$.

When frictional particles $\left(\mu_{s p p} \neq 0\right.$ and $\mu_{r p p} \neq 0$ ) instead of frictionless particles were used for the granular bed, stress jumped (i.e., drastically increased) at the bed surface and then increased linearly with the increase in immersion depth ${ }^{[13,14]}$. That is, interparticle friction caused the initial jump. We labeled the initial jump as $f_{0}$. When friction $\left(\mu_{s p p}\right)$ was high, stress was great, and the stress-increasing number $\left(K_{\sigma}\right)$ and stress jump $\left(f_{0}\right)$ were high (Fig. 3a). Figs. $\mathbf{3 b}$ and $\mathbf{3} \boldsymbol{c}$ show the normal stress at different penetration directions and initial orientations. $f_{\sigma}$ decreased with increasing orientation angle $(\beta) . K_{\sigma}$ and $f_{0}$ at different orientation angles $\beta$ are presented as open squares in Fig. $3 d$. $K_{\sigma}$ was always greater than 1 , and $K_{\sigma}$ and $f_{0}$ decreased gradually with increasing $\beta$. Stress in the frictional granular bed was influenced by the plate orientation but was independent of the penetration direction. The relationship between stress and immersion depth also did not change when the penetration direction $(\gamma)$ was varied (Figs. $\mathbf{3} \boldsymbol{b}$ and $\mathbf{3} \boldsymbol{c}$ ). Notably, the granular beds shown in Fig. $3 \boldsymbol{b}$ were loose with a solid volume fraction $(\zeta)$ of 0.585 . Stress increased when the bed was densely packed before penetration. Fig. $3 c$ shows stress in the packed bed of $\zeta=0.646$. Stress at the bed surface jumped more sharply than that in the loose bed. Although stress and stress jump in the packed bed was considerably greater than that in the loose bed, the relationships between $K_{\sigma}$ and $\beta$ exhibited a similar trend and had similar values (Fig. 3d).

When the plate was frictional, friction can generate shear stress on the plate. The shear stress on a frictional plate was extremely small although it existed when the particles were frictionless. The normal stress on the frictional plate was almost similar to that on the frictionless plate (Fig. 4a). The relationships between $f_{\sigma}$ and $h$ at different $\gamma$ values coalesced into a master curve. The penetration direction barely affected the normal stress on a frictionless bed even when the plate was frictional.

However, if both the particles and plate were frictional, stress first depended on the penetration direction. Figs $4 b$ and $4 c$ show the normal stress on the plate placed horizontally $(\beta=0)$ in loose and densely packed frictional granular beds, respectively. Regardless of the penetration direction, the normal stress at the bed surface suddenly 
jumped and then increased linearly. The normal stress and the corresponding $K_{\sigma}$ and $f_{0}$ decreased with decreasing $\gamma$. A detailed examination of Fig. $4 c$ with Fig. $4 b$ revealed that the packed bed produced greater stress and a sharper initial jump than the loose bed but the relationships between $K_{\sigma}$ and $\gamma$ were similar to one another (Fig. 4d).

When the plate was placed horizontally and penetrated vertically $(\beta=0$ and $\gamma=\pi / 2)$, no shear stress was observed because of geometric symmetry, i.e., $f_{\tau}=0$. Under this condition, stress on the frictional plate was similar to that on the frictionless plate, i.e., the effect of particle-plate friction on stress could be negligible ( $\square$ and $\circ$ in Figs $4 a$ and $4 \boldsymbol{b})$. When penetration was oblique $(\beta=0$ but $\gamma \neq \pi / 2)$, particle-plate friction resulted in shear stress. Fig. 4d shows the equivalent friction coefficient $f_{v} f_{\sigma}$ at different penetration angles. The equivalent friction coefficient $f_{t} / f_{\sigma}$ increased with the reduction in penetration angle, but was still smaller than the specified friction coefficient $\mu_{s p i}\left(\mu_{s p i}=0.4\right)$. $K_{\sigma}$ and $f_{t} f f_{\sigma}$ only increased slightly when the bed was packed densely, whereas $f$ and $f_{0}$ increased considerably.

Besides the penetration direction, the stress also is influenced by the plate orientation of frictional plate. When the plate penetrated vertically $(\gamma=\pi / 2)$, Figs $\mathbf{5} \boldsymbol{a}$ and $\mathbf{5} \boldsymbol{b}$ show the effect of orientation on normal stress on loose and densely packed frictional granular beds, respectively. The oblique plate has smaller stress than that placed horizontally. Fig. $\mathbf{5} \boldsymbol{d}$ presents the relationship between $K_{\sigma}, f_{0}$ and $\beta$. With $\beta$ increasing, both $K_{\sigma}$ and $f_{0}$ decreased, but the equivalent friction coefficient $f_{v} / f_{\sigma}$ increased. Similar with Fig 4 , the packed bed has larger stress and a sharper initial jump than the loosed bed.

Fig. $5 c$ shows the stress on a plate penetrating at $\gamma=\pi / 4$. The plate placed obliquely experienced upward stress for $\beta+\gamma>0(\square, \diamond$ and $\nabla$ in Fig. $\mathbf{5 c}$ ) while downward stress for $\beta+\gamma<0$ ( $\triangle$ in Fig. $\mathbf{5 c}$ ). When the direction of movement was the same as that of orientation $(\beta+\gamma=0)$, the stress is extremely small $\left(f_{\sigma} \approx 0\right)$. While $\beta+\gamma<0$, the upper surface of the plate suffered the stress $\left(f_{\sigma}<0\right)$, namely, the particles were lifted. $K_{\sigma}, f_{0}$ and $f_{t} f_{\sigma}$ have non-monotonic functions with $\beta . K_{\sigma}$ and $f_{0}$ increase first and then decrease with increasing $\beta$, and show maximum value at $\beta=0($ Fig. $5 \boldsymbol{e})$. $\left|f_{t} / f_{\sigma}\right|$ decrease first and then increase with the increase of $\beta$ and presents a maximum at $\beta+\gamma=\pi / 2$ (Fig. 5f).
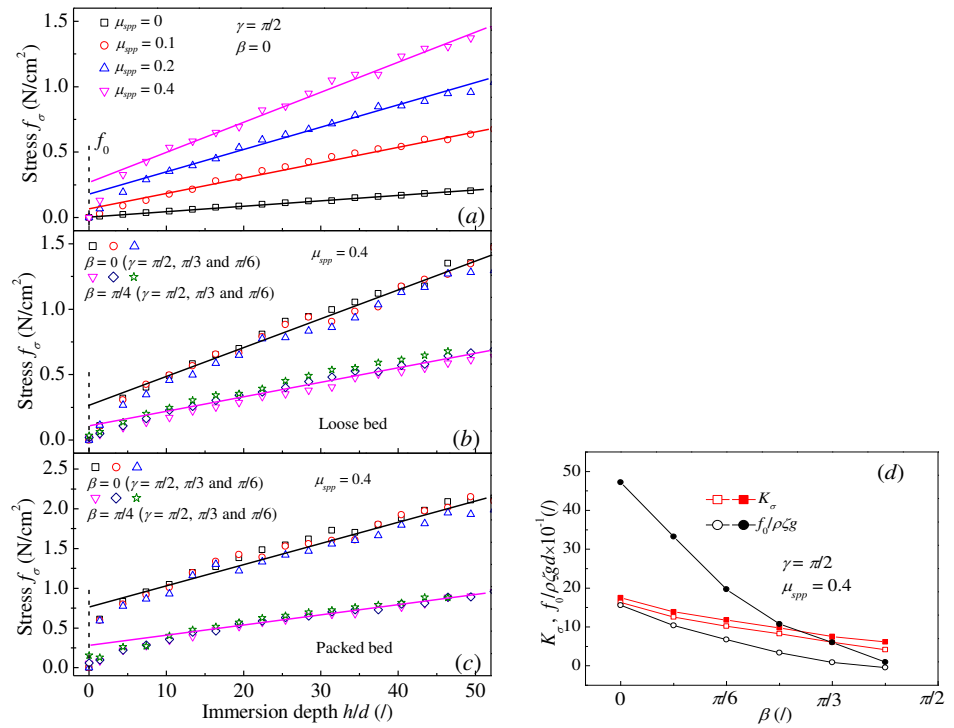

Fig. 3 Stress on the frictionless plate during penetration. $l / d=10, \mu_{s p i}=\mu_{r p i}=0, \mu_{r p p}=0.1$. For the frictional granular bed, stres is linear to the immersion depth but jumps at the bed surface and then increases linearly. Stress jump $f_{0}$ and increasing number $K_{\sigma}$ increase with the increase in $\mu_{s p p}$ as depicted in $(a)$. Stress on the frictionless plate is influenced by $\beta$ but is independent of $\gamma$ as shown by $(b)$ and $(c)$, and stress jump $f_{0}$ and increasing number $K_{\sigma}$ increase with the increase in $\mu_{s p p}$ as depicted in $(d)$. Open symbols in $(d)$ represent the loose bed and solid symbols represent the packed bed. Stress in the packed bed is larger than that in the loose bed. 

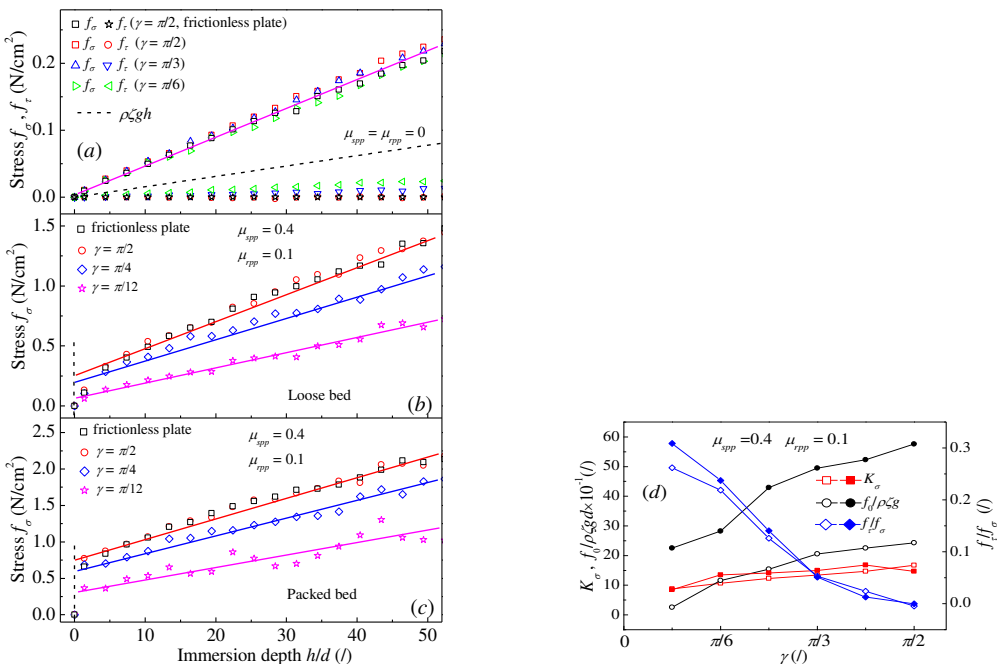

Fig. 4 Effect of penetration direction on stress on frictional plate. $l / d=10, \mu_{s p i}=0.4, \mu_{r p i}=0.1, \beta=0$. Stress on frictional plate is affected by $\gamma$ as illustrated in $(b)$ and $(c) . f_{0}$ and $K_{\sigma}$ increase with the increase in $\gamma$. In addition to normal stress $f_{\sigma}$, shear stress $f_{\tau}$ exists on the frictional plate, and $f_{\tau} / f_{\sigma}$ decreases with $\gamma$ increasing, as depicted in $(d)$. Open symbols in $(d)$ represent the loose bed and solid symbols represent the packed bed. Stress in the packed bed is larger than that in the loose bed.
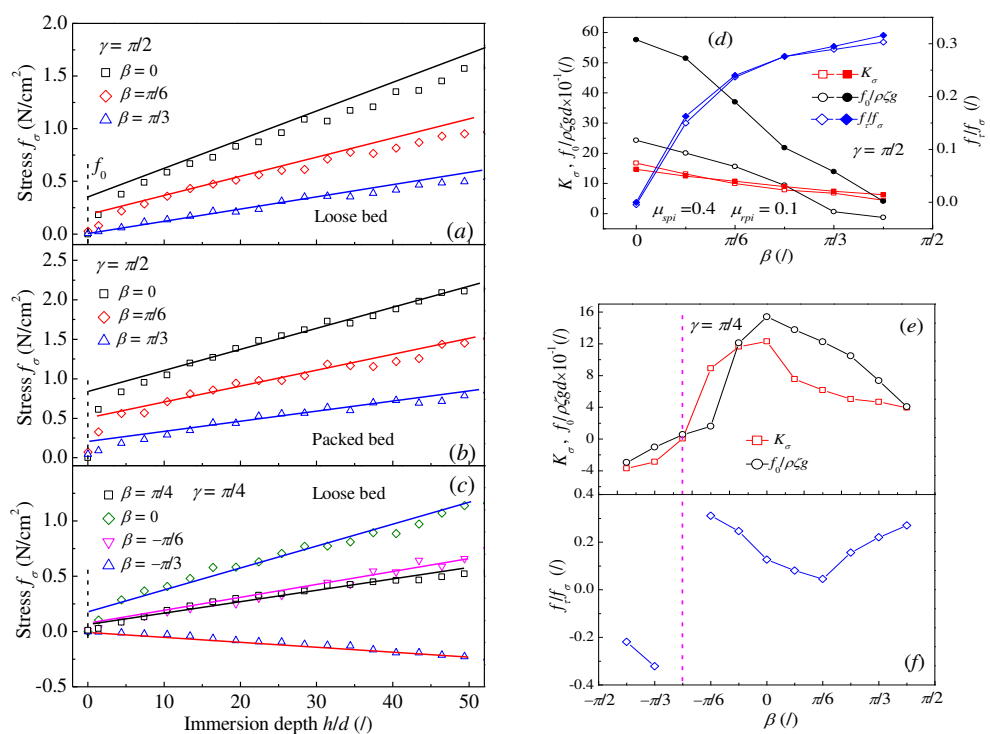

Fig. 5 Effect of plate orientation on stress on frictional plate. $l / d=10, \mu_{s p p}=\mu_{s p i}=0.4, \mu_{r p p}=\mu_{r p i}=0.1$. Stress on plate penetrating vertically is affected by $\beta$ as illustrated in $(a),(b)$ and $(d) . f_{0}$ and $K_{\sigma}$ increase but $f_{\tau} / f_{\sigma}$ decreases with the increase in $\beta$ for vertical penetration. Open symbols in $(d)$ represent the loose bed and solid symbols represent the packed bed. $(c),(e)$ and $(f)$ present the stress of oblique penetration. Downward stress can exist when $\beta+\gamma<0$, as shown in $(c)$; and $f_{0}, K_{\sigma}$ and $f_{\tau} / f_{\sigma}$ show non-monotonic function with $\beta$ in $(e)$ and $(f)$. 


\section{Particle motion}

The stress on the plate mainly originates from the resistance of the contacting particles. Because of the block of bed bottom, the particles cannot always move downward and must change their movement direction. Fig. 6 shows the velocity profile of particles in the granular bed induced by the plate. The friction of the particles played an important role in their motion. If the particles were frictionless, then only the particles around the plate were displaced (Fig. 6a ). For a frictional granular bed, most particles were pushed away (Fig. $\boldsymbol{6} \boldsymbol{b}$ ) and moved at a higher velocity (Figs $\mathbf{6} \boldsymbol{c}-\mathbf{6} f$ ). The average velocity in computed domain, $v_{p, a}$, was 1.75 for frictionless particles (Figs $6 \boldsymbol{a}$ ), while 2.33 and 2.15 for frictional particles (Figs $\mathbf{6} \boldsymbol{c}, \boldsymbol{6} \boldsymbol{e}$ ), respectively. Therefore, the plate suffered from a higher resistance in the frictional bed than in the frictionless bed.

The particle velocity profile was influenced by the direction of plate movement (i.e., the penetration direction) when the plate was frictional. However, it was negligibly affected when the plate was frictionless. No shear force was present between the plate and the particles. Thus, the particles that were in contact with the frictionless plate exhibited similar movement regardless of penetration direction (Figs. $\mathbf{6} \boldsymbol{c}$ and $\mathbf{6} \boldsymbol{d}$ ). Therefore, stress was independent of penetration direction (Figs. $\mathbf{3} \boldsymbol{b}$ and $\mathbf{3 c}$ ). By contrast, if the plate was frictional, particle motion was influenced by the penetration direction of the plate (Figs. $6 \boldsymbol{e}$ and $\mathbf{6} f$ ). Moreover, the effect of particle-plate friction could be neglected when the plate was penetrating vertically but it could not be ignored in oblique penetration. In vertical penetration $(\gamma=\pi / 2)$, the particle-plate friction barely influenced particle movement, and similar velocity profiles were obtained for frictionless and frictional plates (Figs. $\mathbf{6} \boldsymbol{c}$ and $\mathbf{6} \boldsymbol{e}$ ). When the plate was moved obliquely $(\gamma \neq \pi / 2$ ), the velocity profiles induced by the frictionless and frictional plates were different (Figs. $\mathbf{6} \boldsymbol{d}$ and $\mathbf{6} f$ ).

Furthermore, particle motion was affected by plate orientation. Figs $\boldsymbol{6} \boldsymbol{e}$ and $\boldsymbol{\sigma} \boldsymbol{g}$ show the particle velocity profiles during penetration at the same penetration angle but different orientations. The particles moved to the side of the head-on surface during vertical penetration when the plate was inclined (Fig. 6g). Apparently, the movement direction of particles is determined by the plate movement direction as well as the orientation. The particles moved following the plate's movement at most times (Figs $\mathbf{6} \boldsymbol{c}-\mathbf{6 g}$ ), but sometimes can be pushed away to the opposite side of the plate's movement when the orientation angle $\beta<0$ (Fig. $6 \boldsymbol{h}$ ).

For a plate placed horizontally and penetrated vertically, the particles showed approximately symmetric field (Fig. $\boldsymbol{6} \boldsymbol{b}$, Figs $\mathbf{6} \boldsymbol{a}, \boldsymbol{6} \boldsymbol{c}$ and $\boldsymbol{6} \boldsymbol{e}$ ). If the plate was frictionless, the penetration direction hardly affected the particles' velocity field; therefore, stress on frictionless plate was not influenced by $\gamma$ (Figs $\mathbf{4 b}$ and $\mathbf{4 c}$ ). For the fractional plate, both $\beta$ and $\gamma$ can bring in asymmetry field (Figs $\mathbf{6} \boldsymbol{f}-\mathbf{6} \boldsymbol{h}$ ); therefore, stress on frictional plate was affected by $\beta$ and $\gamma$ (Figs. 4 and 5).

We defined the angle between the movement direction of the contacting particles and the horizontal direction as the push angle $(\alpha)$ for describing the motion of the particles quantitatively. When the plate was frictionless, the particles moved along the normal direction of the plate and angle $\alpha$ was only affected by $\beta$. When the plate was frictional, the particle movement direction was between the normal and penetration directions of the plate, and angle $\alpha$ was influenced by $\beta$ and $\gamma$. The push angle could be adopted to estimate stress on the plate. When the particles moved downward, $\alpha=\pi / 2$, stress had a maximum value and decreased with the increase in $\alpha$. The relationship between stress and push angle will be further discussed in section 5 . 


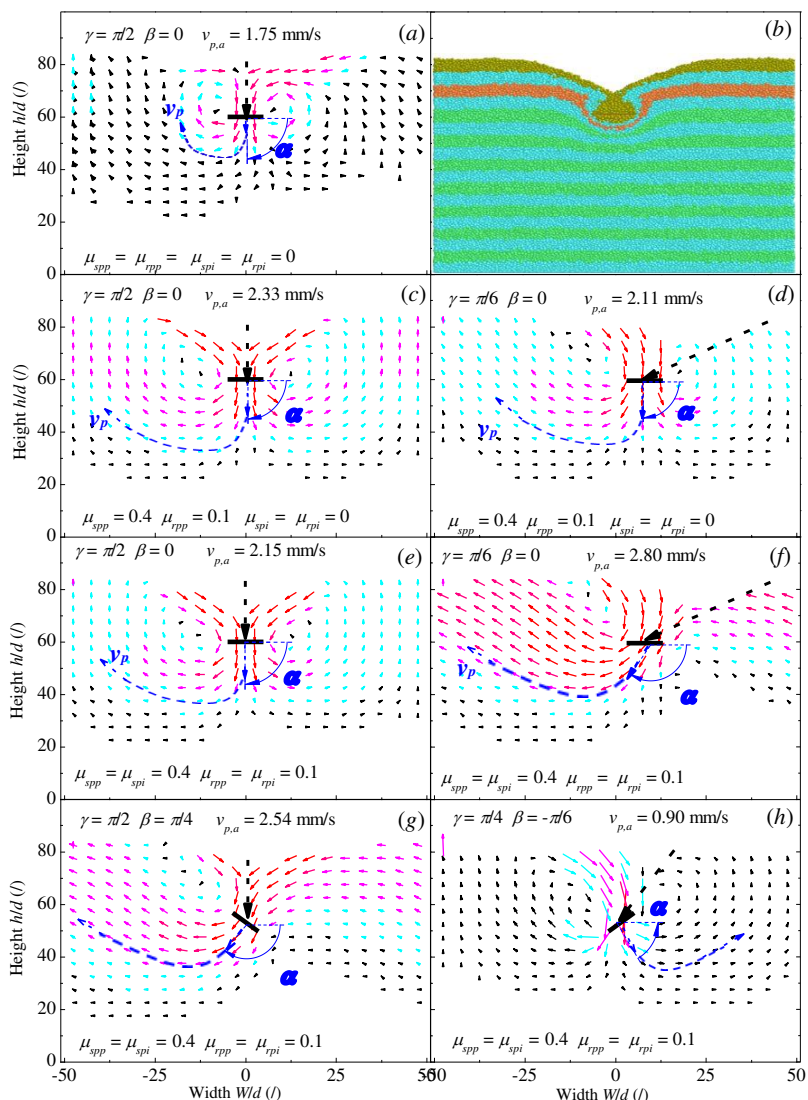

Fig. 6 Velocity profiles during penetration with $l / d=10$ and $v=20 \mathrm{~mm} / \mathrm{s}$. The red, pink, magenta, cyan and black vectors represent the velocity of particles $\left(v_{p}\right)$ in the range of $v_{p}>8 \mathrm{~mm} / \mathrm{s}, 6 \mathrm{~mm} / \mathrm{s}<v_{p}<8 \mathrm{~mm} / \mathrm{s}, 4 \mathrm{~mm} / \mathrm{s}<v_{p}<6 \mathrm{~mm} / \mathrm{s}, 2 \mathrm{~mm} / \mathrm{s}<v_{p}<4$ $\mathrm{mm} / \mathrm{s}$, and $v_{p}<2 \mathrm{~mm} / \mathrm{s}$, respectively. $v_{p, a}$ is the average velocity of particles in bed induced by plate penetration. The blue dotted lines guide the movement of the particles. (a) shows the velocity profile of a frictionless bed, and only the particles around the plate move. A lot of particles are pushed away if the particles are frictional as shown in $(b)$. The velocity profile is influenced by the direction of plate penetration and orientation when the plate is frictional but is barely affected by penetration direction when the plate is frictionless. $(c)$ and $(d)$ show the velocity profile of a frictional bed but with a frictionless plate; and $(e),(f),(g)$ and $(h)$ show the velocity profile of a frictional bed and a frictional plate.

\section{Theoretical analysis}

The particles in front of the plate moved as the plate penetrated, and their motion exhibited a different direction because of slippage between the plate and the particles. Based on previous research ${ }^{[13,21]}$ and the bearing capacity theory of soil mechanics, ${ }^{[22]}$ the slip boundary was formed by shearing, as indicated by the curve BOCD in Fig. 7a $\mathrm{BO}$ and $\mathrm{CD}$ were straight lines, and $\mathrm{OC}$ was a logarithmic spiral that was tangent to lines $\mathrm{BO}$ and $\mathrm{CD}$ at points $\mathrm{O}$ and $\mathrm{D}$, respectively. The movement of the particles could be divided into three zones: Zone I was a plastic zone, AOB Zone II was a fan zone, OAC; and Zone III was a passive zone, CAD. The angle of $\angle \mathrm{AOB}$ remained constant during plate movement, whereas the geometric shape of Zone I was influenced by the orientation direction $(\beta)$ and movement direction $(\gamma)$ of the plate. In Zone III, the angle between DC and the horizontal direction $(\angle \mathrm{CDA})$ was equal to $\pi / 4$ $-\varphi / 2$. Curve $\mathrm{AD}$ suffered from uniform stress that was equal to the hydrostatic-like pressure from the above layer, $f_{A D}=\rho \zeta g h$. Zone II was a transition zone connecting Zones I and III. The logarithmic spiral OC followed $l_{A P}=$

$22 l_{A O e^{\psi t a n} \varphi}$, where $\varphi$ is the internal friction angle of the particles, $P$ is a point on curve $\mathrm{OC}, \psi$ is the angle of $\angle \mathrm{OAP}$, and 
$l_{A P}$ and $l_{A O}$ are the lengths of lines AP and AO.

The push angle $\alpha$ (also the angle between the movement direction of the particles in Zone I and the horizontal direction) is influenced by $\beta$ and $\gamma$. We assumed that $\alpha$ can be estimated by using the following expression ${ }^{[18] \text { : }}$

$$
\alpha=\xi(\pi-\gamma)+(1-\xi)(\beta+\pi / 2 \pm \pi) \text {, }
$$

where " - " is for $\beta+\gamma \geq 0$, which denotes that the downward side of the plate is the head-on face; and " + " is for $\beta$ $+\gamma<0$, denoting that the upward side is the head-on face. $\xi$ is a proportion factor and related to particle-plate friction. $\xi$ is between 0 and 1 , and increases with the particle-plate friction increasing. $\xi=0$ indicates that the plate is frictionless, and $\xi=1$ indicates an absolute frictional plate. In our discussion, $\alpha$ changed from $-\pi / 2$ to $3 \pi / 2$ as $\beta$ and $\gamma$ ranged from $-\pi / 2$ to $\pi / 2$ and 0 to $\pi$, respectively. In the following, we only analyzed the force on the plate in detail when $\alpha>\pi / 2$, and the force of $\alpha<\pi / 2$ could be computed on the basis of symmetry as $f_{z}(\alpha, \beta)=f_{z}(\pi-\alpha,-\beta), f_{x}(\alpha, \beta)$ $=-f_{x}(\pi-\alpha,-\beta)$

Three different conditions existed with the change in $\alpha$ and $\beta$ as shown in Figs. 7a-7c. $\angle \mathrm{AOB}, \angle \mathrm{OBA}$, and $\angle \mathrm{CAD}$ are marked as $\eta, \chi$, and $\theta$, respectively. The angle of $\angle \mathrm{OBA}(\chi)$ could be obtained by analyzing the geometric relation in Fig. 7a as $\chi=3 \pi / 2 \pm \pi / 2-\alpha+\beta$, where "-" is for $\beta+\gamma \geq 0$ and "+" is for $\beta+\gamma<0$. Zone I existed when $\chi<\pi$. According to Terzaghi theory ${ }^{[22]}, \eta \equiv \pi / 2-\varphi$ for $\chi<\pi / 2+\varphi$, and $\eta=\pi-\chi$ for $\pi / 2+\varphi \leq \chi<\pi$. The angles of $\angle \mathrm{OAB}$ and $\angle \mathrm{OAC}$ exhibited the following relationship: $\angle \mathrm{OAB}=\pi-\chi-\eta$ and $\angle \mathrm{OAC}=\pi+\eta-\theta-\alpha$. Zone I did not exist when $\chi \geq \pi$ as shown in Fig. $7 \boldsymbol{b}$; $\mathrm{AO}$ was coincident with $\mathrm{AB}$ and $\eta=0$. Zone II existed when $\angle \mathrm{OAD}>$ $\angle \mathrm{CDA}(\pi+\eta-\alpha>\pi / 4-\varphi / 2)$. In accordance with Terzaghi theory ${ }^{[22]}$, the angle of $\angle \mathrm{CAD}$ was equal to $\pi / 2-\varphi(\theta$ $=\pi / 2-\varphi)$. Zone II did not exist when $\angle \mathrm{OAD} \leq \angle \mathrm{CDA}(\pi+\eta-\alpha \leq \pi / 4-\varphi / 2)$ as shown in Fig. $7 c$, and $\theta=3 \pi / 4+$ $\eta-\alpha-\varphi / 2$.

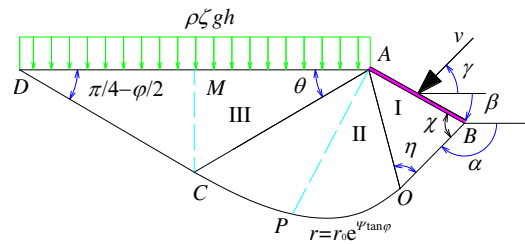

(a)

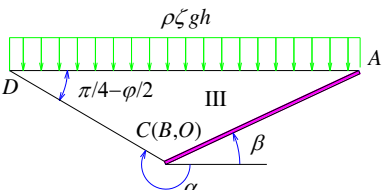

(c)

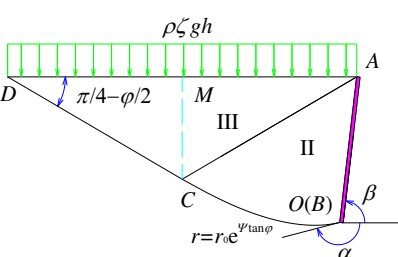

(b)

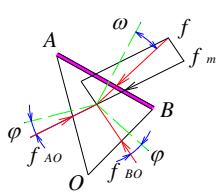

(d)

Fig. 7 Force analysis of the plate in a bed. (a) Particle motion induced by penetration can be divided into three zones, namely, Zones I, II, and III. The force balance of Zone I is shown in $(d)$. Zone I does not exist when $\angle A B O \geq \pi(b)$, and Zone II does not exist when $\angle \mathrm{OAD} \leq \angle \mathrm{CDA}(c)$

The lengths of curves $\mathrm{AO}, \mathrm{AC}, \mathrm{CM}$, and $\mathrm{AM}$ are expressed as

$$
\begin{gathered}
l_{A O}=l(\cos \angle \mathrm{OAB}+\sin \angle \mathrm{OAB} \cot \eta), \\
l_{A C}=l_{A O e^{\angle \mathrm{OACtan} \varphi},} \\
l_{C M}=l_{A C \sin \theta,} \\
l_{A M}=l_{A C} \cos \theta .
\end{gathered}
$$

Considering Coulomb's law, the stress on CM can be expressed as

$$
f_{C M}=\rho \zeta g h \cot ^{2}(\pi / 4-\varphi / 2)
$$

$\mathrm{CO}$ is a logarithmic spiral and the stress on $\mathrm{CO}$ is complex. Here, we consider that the average shear stress, $\tau_{\mathrm{CO}}$, is simply proportional to the pressure from the above particles and can be estimated by the following expression,

$$
\tau_{\mathrm{CO}}=k \mu_{\text {spp }} \rho \zeta g\left(h+l_{C M}\right),
$$

where $k$ is the scale factor and $\mu_{s p p}$ is the friction coefficient.

The torque balance of point A can be expressed as follows:

$$
M_{A M}+M_{C M}+M_{C O}=M_{A O},
$$




$$
\begin{aligned}
& M_{A M}=0.5 f_{A D} l_{A M^{2}}, \\
& M_{C M}=0.5 f_{C M} l_{C M^{2}} \text {, } \\
& M_{A O}=0.5\left(f_{A O} \cos \varphi\right) l_{A O}{ }^{2}, \\
& M_{C O}=\int \tau_{\mathrm{CO}} l_{A P}{ }^{2} d \psi=0.5 \tau_{\mathrm{CO}} l_{A O}{ }^{2} \cot \varphi\left[e^{2 \angle \mathrm{OACtan} \varphi}-1\right],
\end{aligned}
$$

where $M_{A M}, M_{C M}, M_{C O}$, and $M_{A O}$ are the torque on the curves of AM, CM, CO, and AO, respectively.

The forces on the curves of $\mathrm{AB}, \mathrm{AO}$, and $\mathrm{BO}$ were balanced, as shown in Fig. $7 \boldsymbol{d}$, where $f, f_{A O}$, and $f_{B O}$ are stresses on $\mathrm{AB}, \mathrm{AO}$ and $\mathrm{BO}$, respectively. On the basis of force balance, stress on the plate $f$ can be obtained as follows:

$$
\begin{gathered}
f_{A O} l_{A O}=f_{m} l, \\
f=f_{m}[\cos (\angle \mathrm{OAB}-\varphi-\omega)+\sin (\angle \mathrm{OAB}-\varphi-\omega) \tan (\pi / 2+(\angle \mathrm{OAB}-\varphi-\omega)-(\pi-\eta-2 \varphi))],
\end{gathered}
$$

where $\omega$ is the intersection angle between stress $f$ and the normal direction of the plate. $\omega=\angle \mathrm{OAB}-\varphi$ when $-\varphi_{p i}<$ $\angle \mathrm{OAB}-\varphi<\varphi_{p i} ; \omega=\varphi_{p i}$ when $\angle \mathrm{OAB}-\varphi>\varphi_{p i}$; and $\omega=-\varphi_{p i}$ when $\angle \mathrm{OAB}-\varphi<\varphi_{p i}$, where $\varphi_{p i}$ is the friction angle of the plate.

Stress on the plate $\mathrm{AB}$ can be solved by coupling Equations 3-7, as follows:

$$
f=K_{A} K_{B} \rho \zeta g h+K_{A} f_{m g}
$$

where the first item on the right is stress induced by the hydrostatic pressure of the granular bed above the plate. $K_{A}$ is the stress transition coefficient in Zone I, and $K_{B}$ is the stress transition coefficient in Zones II and III.

$$
K_{A}=[\cos (\pi-\chi-\eta)+\sin (\pi-\chi-\eta) \cot \eta]
$$$$
[\cos (\pi-\chi-\eta-\varphi+\omega)+\sin (\pi-\chi-\eta-\varphi+\omega) \tan (\pi / 2-\chi-\omega+\eta)] / \cos \varphi
$$$$
K_{B}=\left[1+\cot ^{2}(\pi / 4-\varphi / 2)\right] e^{2(\eta+\alpha-\theta) \tan \varphi} \sin ^{2} \theta+k \mu_{s p p} \cot \varphi\left(e^{2(\eta+\alpha-\theta) \tan \varphi-1)} .\right.
$$

And $f_{m g}$ is the shear stress induced by the friction and gravity of particles.

$$
f_{m g}=k \mu_{s p p \rho} \zeta g l_{A O} e^{(\eta+\alpha-\theta) \tan \varphi} \sin \theta \cot \varphi\left(e^{2 \angle O A C \tan \varphi}-1\right) .
$$

Normal and shear stresses can be expressed as $f_{\sigma}=f \cos \omega$ and $f_{\tau}=f \sin \omega$, respectively, i.e., $f_{\tau} / f_{\sigma}=\tan \omega$. Therefore,

$$
\begin{array}{ll}
\text { where } & f_{\sigma}=K_{\sigma} \rho \zeta g h+f_{0}, \\
\text { and } & K_{\sigma}=K_{A} K_{B} \cos \omega, \\
f_{0} & =K_{A} f_{m g} \cos \omega .
\end{array}
$$

When the particles were frictionless $\left(\mu_{s p p}=0\right), f_{\sigma}=K_{\sigma} \rho \zeta g h$. Stress was directly proportional to the immersion depth $h$ in a frictionless bed. For frictional particles, $\mu_{s p p} \neq 0$ and $f_{\sigma \mid h \rightarrow 0}=f_{0}$, resulted in a stress jump at the bed surface $(h \rightarrow 0)$.
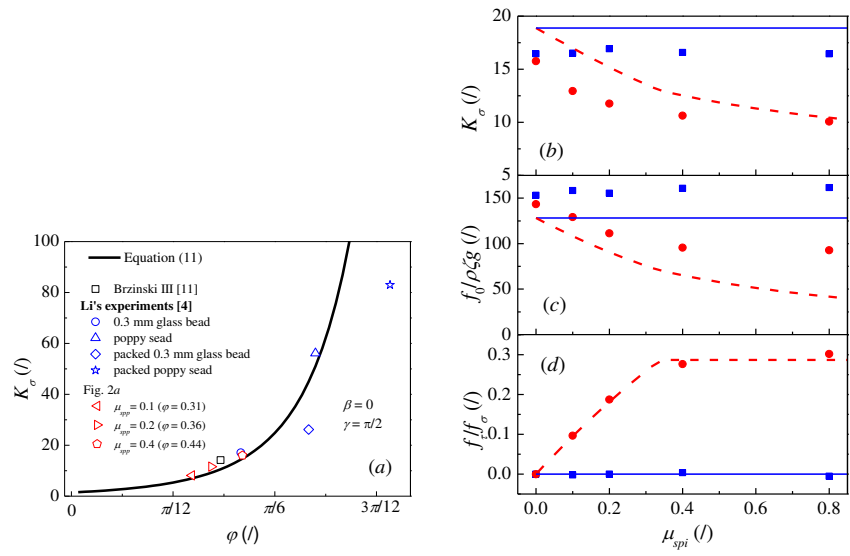

Fig. 8 Theoretical calculation of stress. (a) Effect of $\varphi$ on $K_{\sigma} . K_{\sigma}$ increases with the increase in $\varphi .(b),(c)$, and $(d)$ Effect of particle-plate friction, $\beta=0, \varphi=\pi / 6, \varphi_{p i}=0.28, \mu_{s p p}=0.4, \mu_{r p p}=0.1, \mu_{r p i}=0.1$, and it is assumed that $\xi=1-\exp \left(-\mu_{s p i}\right)$. Stress is influenced by penetration direction when the plate is frictional. The solid square scatters and solid lines are for $\gamma=\pi / 2$, and the solid circular seatters and dotted lines are for $\gamma=\pi / 6$. The solid scatters represent the results of DEM simulation, and the lines are calculated in accordance with theoretical analysis.

Fig. $8 \boldsymbol{a}$ shows the relationship between $K_{\sigma}$ and $\varphi$ when the plate was penetrating vertically. $K_{\sigma}$ increased rapidly with the increase in $\varphi$. The results of our model were qualitatively consistent with these in previous experiments ${ }^{[5,12]}$ 


\begin{abstract}
and our DEM simulations. For the DEM simulation, $\varphi$ was estimated in accordance with the shear test ${ }^{[18]}$. In general, $\varphi$ increased with increasing particle friction, and then stress increased; furthermore, all particle parameters relevant to the internal frictional angle could increase stress and cause the jump.

Figs. $8 \boldsymbol{b}-\mathbf{8} \boldsymbol{d}$ show the effect of $\mu_{s p i}$ on stress on the penetrating plate. If the plate was frictionless $\left(\mu_{s p i}=0\right)$, no shear stress occurred on the plate $\left(f_{\tau}=0\right)$, and $\alpha$ was influenced only by $\beta$ but was independent of $\gamma$. That is to say, stress was independent of the movement direction. $K_{\sigma}, f_{0}$, and $f_{\tau} / f_{\sigma}$ had the same values for different $\gamma$ when $\mu_{s p i}=0$. However, when the plate was frictional $\left(\mu_{s p i} \neq 0\right), K_{\sigma}, f_{0}$, and $f_{\tau} / f_{\sigma}$ were influenced by $\gamma . K_{\sigma}$ and $f_{0}$ decreased with the increase in $\mu_{s p i}$ when $\gamma=\pi / 6$, and $f_{v} f_{\sigma}$ increased and then remained constant. In addition, friction had no effect on stress when the plate was placed horizontally and penetrated vertically, i.e., $\alpha \equiv \pi / 2$. As shown in Figs. 8b-8d, $K_{\sigma}, f_{0}$, and $f_{t} / f_{\sigma}$ remained constant for the frictionless plate with the increase in $\mu_{p i}$. Quantitatively, $K_{\sigma}$ from the theoretical analysis was slightly larger than that from DEM simulation, while $f_{0}$ was relatively smaller. These deviations were induced by the estimation of push angle (Eq. 2). The proportion factor $\xi$ in Eq. 2 is related to the friction between plate and particles, and the relation between $\xi$ and $\mu_{s p i}$ should be further developed in following studies.
\end{abstract}

\title{
6. Conclusions
}

The stress on a thin plate penetrating into a granular bed was studied by DEM simulation and theoretical analysis by changing the penetration and orientation directions. Based on the results, the following conclusions were confirmed.

For frictionless particles, stress on plate was always greater than the hydrostatic pressure of the granular bed and increased linearly with the increase in immersion depth. When frictional particles instead of frictionless particles, a stress jump existed at the surface of granular bed. Both the stress and stress jump increased with friction increasing. For frictionless plate, stress in the frictional granular bed was influenced by the plate orientation but was independent of the penetration direction. Both the stress and jump decreased gradually with increasing orientation angle. For frictional plate, stress in the frictional granular bed was influenced by both plate orientation and penetration direction. The plate placed obliquely experienced upward stress for $\beta+\gamma>0$ while downward stress for $\beta+\gamma<0$.

A theoretical model was presented to calculate the stress on plate. In our model, we demonstrated the reason why friction, penetration direction, and plate orientation affected stress on the plate surface. Stress largely depended on the internal frictional angle, particle push angle, and penetration height. For a frictionless plate, stress was affected by plate orientation but was independent of penetration direction. When the plate was frictional, the plate orientation and penetration directions could affect stress. Stress was essentially determined by the particles' push angle $\alpha$, and the plate orientation and penetration directions influenced stress by changing the particles' push angle $\alpha$. In addition, the effect of friction is typically not considered in most existing models ${ }^{[11-14]}$. On the basis of our results, these models are valid for vertical penetration. Comparatively, particle parameters and plate features are considered in the model, which has extensive applications, including oblique penetration.

\section{Supplementary material}

See supplementary material for the Hertz-Mindlin model, push angle validation and internal friction angle estimation of used particles according to the shearing test.

\section{Data availability}

The data that supports the findings of this study are available within the article [and its supplementary material].

\section{References}

[1] Jaeger, H. M., Nagel, S. R., \& Behringer, R. P. (1996). Granular solids, liquids, and gases. Reviews of modern physics, 68(4), 1259 .

[2] Peng, Z., Zhou, J., Zhou, J., Miao, Y., Cheng, L., Jiang, Y., \& Hou, M. (2021). External pressure dependence of granular orifice flow: Transition to Beverloo flow. Physics of Fluids, 33(4), 043313.

[3] Lohse, D., Rauhe, R., Bergmann, R., \& Van Der Meer, D. (2004). Creating a dry variety of quicksand. Nature, 432(7018), 689-690.

[4] Katsuragi, H., \& Durian, D. J. (2007). Unified force law for granular impact cratering. Nature physics, 3(6), 420423.

[5] Li, C., Zhang, T., \& Goldman, D. I. (2013). A terradynamics of legged locomotion on granular media. Science, 339(6126), 1408-1412.

[6] Meirion-Griffith, G., \& Spenko, M. (2010, March). An empirical study of the terramechanics of small unmanned ground vehicles. In 2010 IEEE Aerospace Conference (pp. 1-6). IEEE. 
[7] Pierce, F. J., Fortin, M. C., \& Staton, M. J. (1994). Periodic plowing effects on soil properties in a no - till farming system. Soil Science Society of America Journal, 58(6), 1782-1787.

[8] Bransby, M. F., Brown, M., Hatherley, A., \& Lauder, K. (2010). Pipeline plough performance in sand waves. Part 1: model testing. Canadian Geotechnical Journal, 47(1), 49-64.

[9] Towarek, Z. (2003). Dynamics of a single-bucket excavator on a deformable soil foundation during the digging of ground. International Journal of Mechanical Sciences, 45(6-7), 1053-1076.

[10] Askari, H., \& Kamrin, K. (2016). Intrusion rheology in grains and other flowable materials. Nature materials, $15(12), 1274-1279$.

[11] Zhang, T., \& Goldman, D. I. (2014). The effectiveness of resistive force theory in granular locomotion. Physics of Fluids, 26(10), 101308.

[12] Brzinski III, T. A., Mayor, P., \& Durian, D. J. (2013). Depth-dependent resistance of granular media to vertical penetration. Physical review letters, 111(16), 168002.

[13] Kang, W., Feng, Y., Liu, C., \& Blumenfeld, R. (2018). Archimedes' law explains penetration of solids into granular media. Nature communications, 9(1), 1-9.

[14] Feng, Y., Blumenfeld, R., \& Liu, C. (2019). Support of modified Archimedes' law theory in granular media. Soft matter, 15(14), 3008-3017.

[15] Zheng, H., Wang, D., Chen, D. Z., Wang, M., \& Behringer, R. P. (2018). Intruder friction effects on granular impact dynamics. Physical Review E, 98(3), 032904.

[16] Kim, S., \& Kamrin, K. (2020). Power-law scaling in granular rheology across flow geometries. Physical Review Letters, $125(8), 088002$.

[17] Bhateja, A., \& Khakhar, D. V. (2020). Analysis of granular rheology in a quasi-two-dimensional slow flow by means of discrete element method based simulations. Physics of Fluids, 32(1), 013301

[18] Supplementary material.

[19] Albert, R., Pfeifer, M. A., Barabási, A. L., \& Schiffer, P. (1999). Slow drag in a granular medium. Physical review letters, 82(1), 205.

[20] Costantino, D. J., Bartell, J., Scheidler, K., \& Schiffer, P. (2011). Low-velocity granular drag in reduced gravity. Physical Review E, 83(1), 011305.

[21] Meyerhof, G. G. (1951). The ultimate bearing capacity of foudations. Geotechnique, 2(4), 301-332

[22] Terzaghi, K. (1943). Theorectical Soil Mechanics, Wiley, New York, NY. 

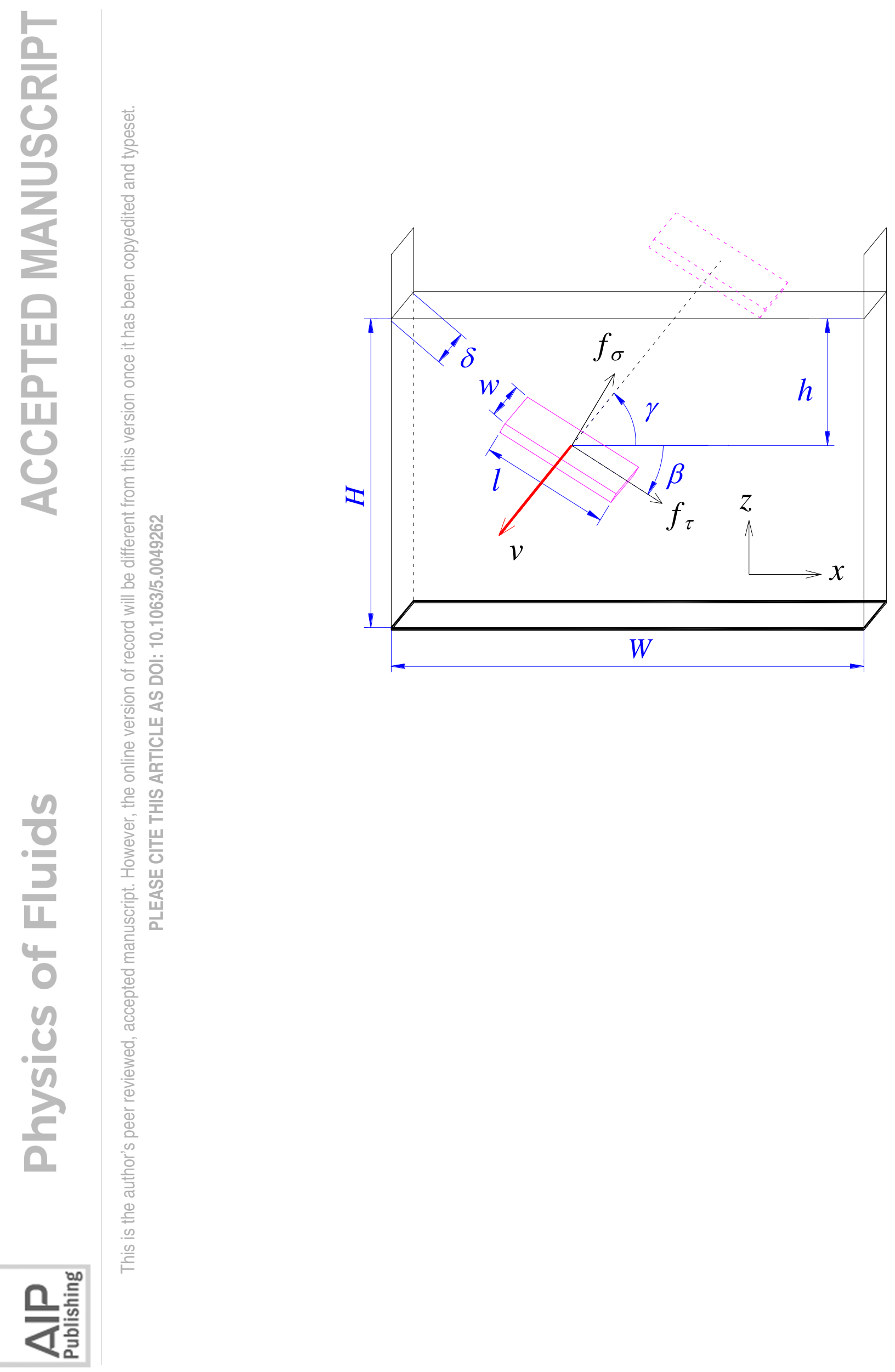


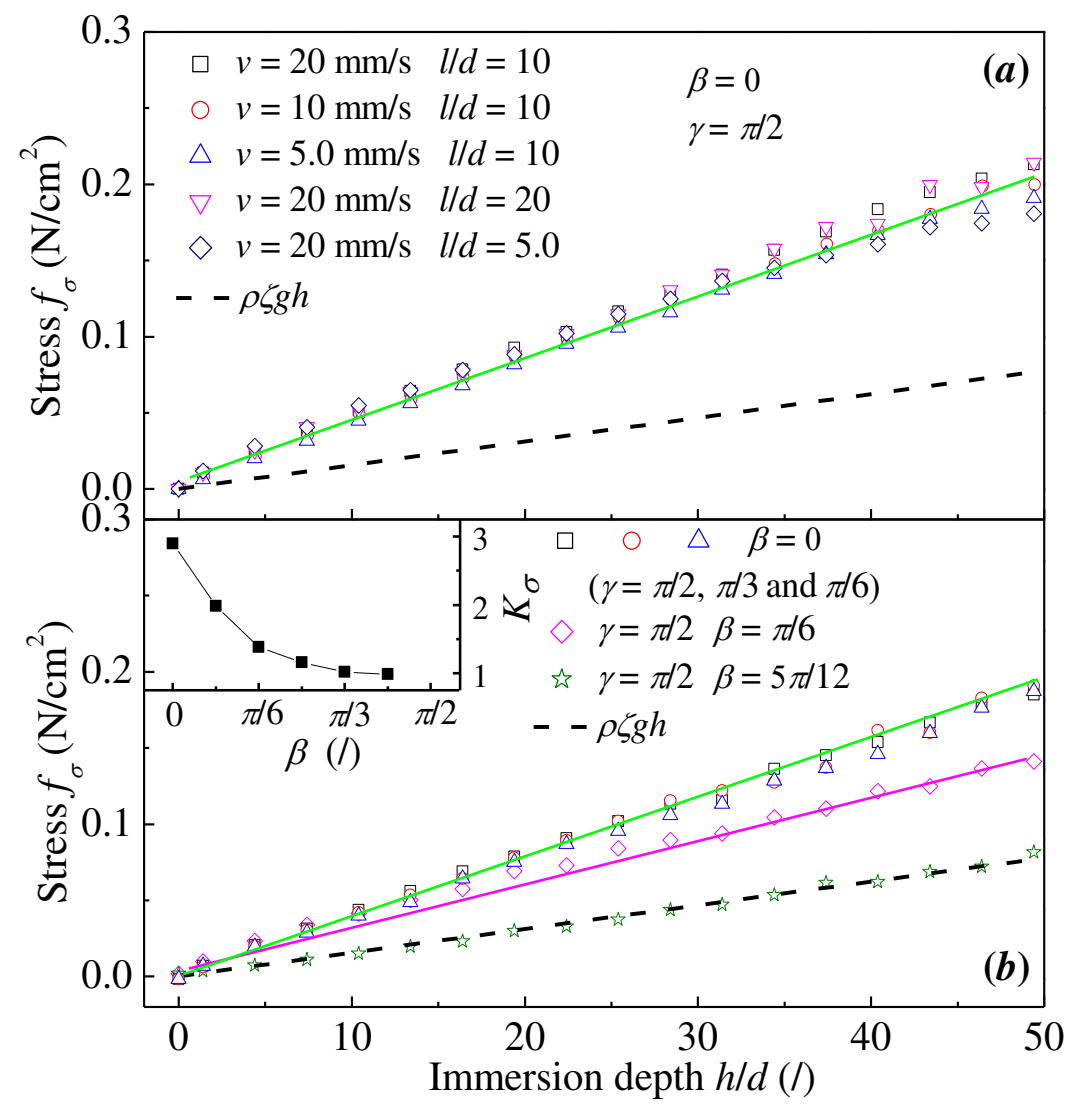




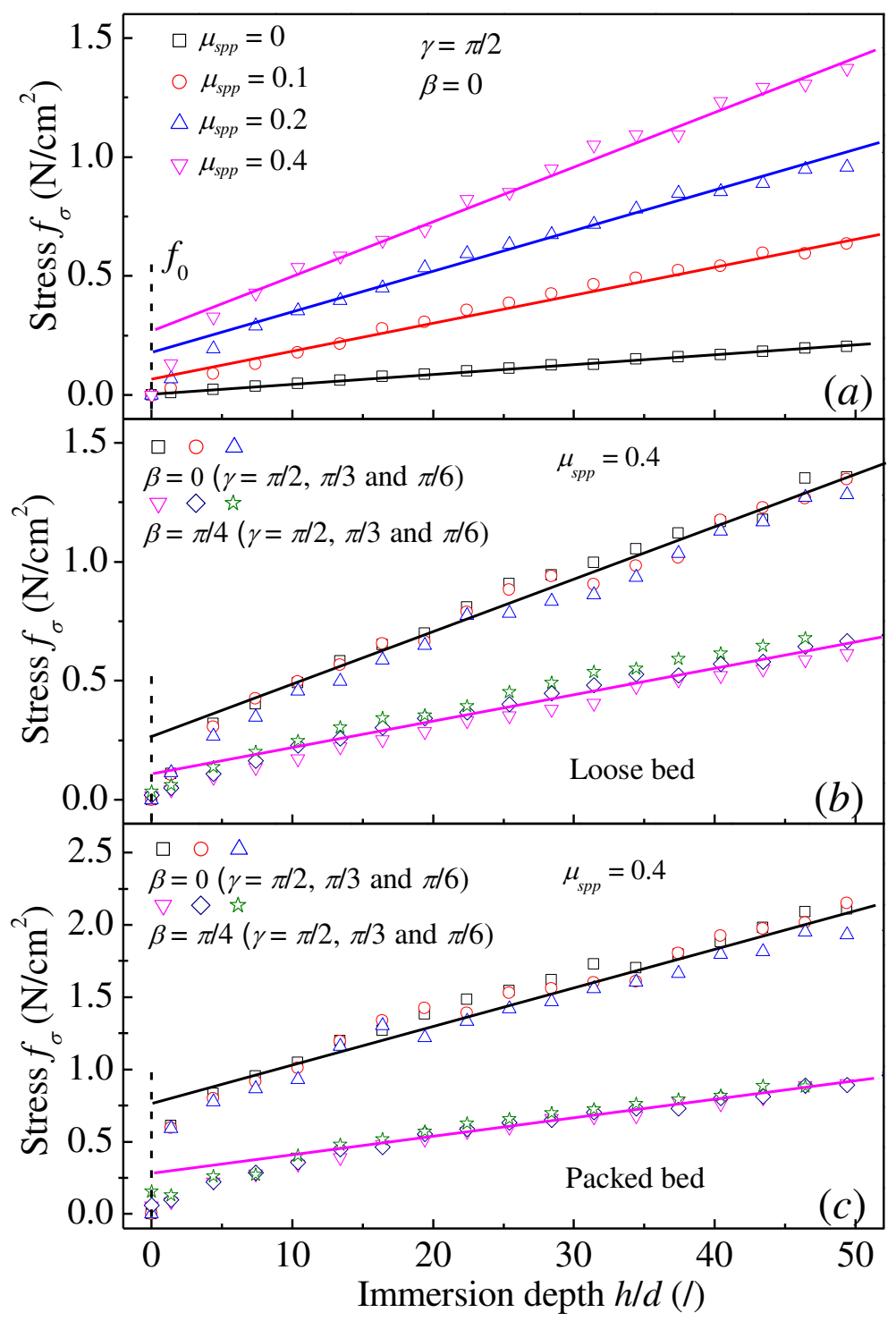




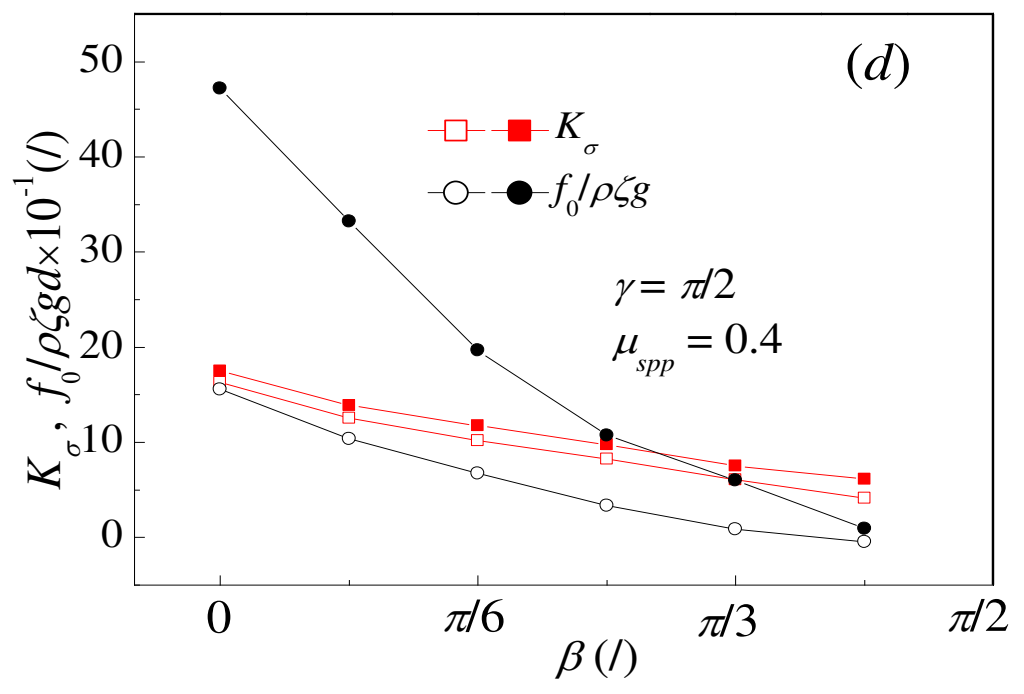




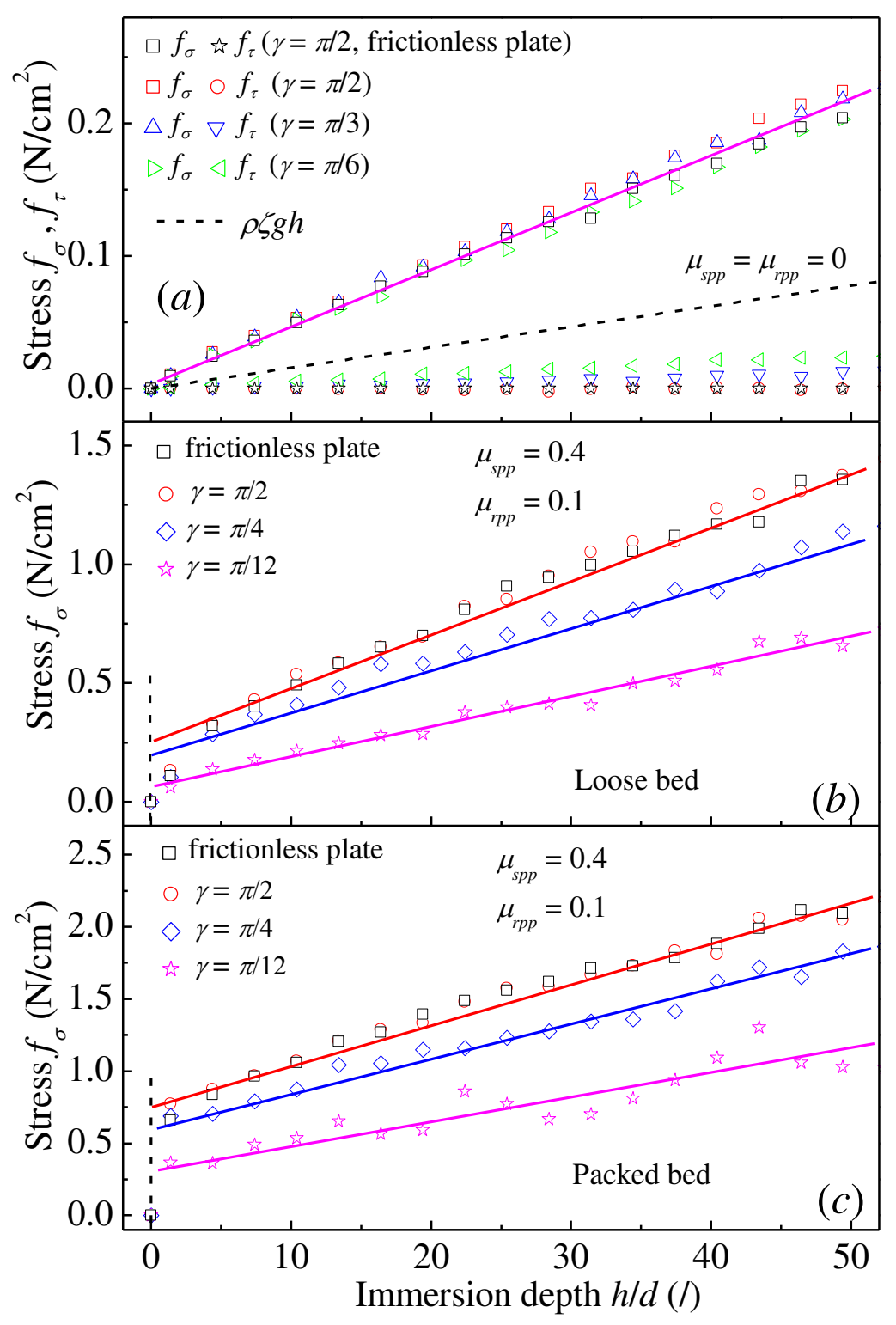




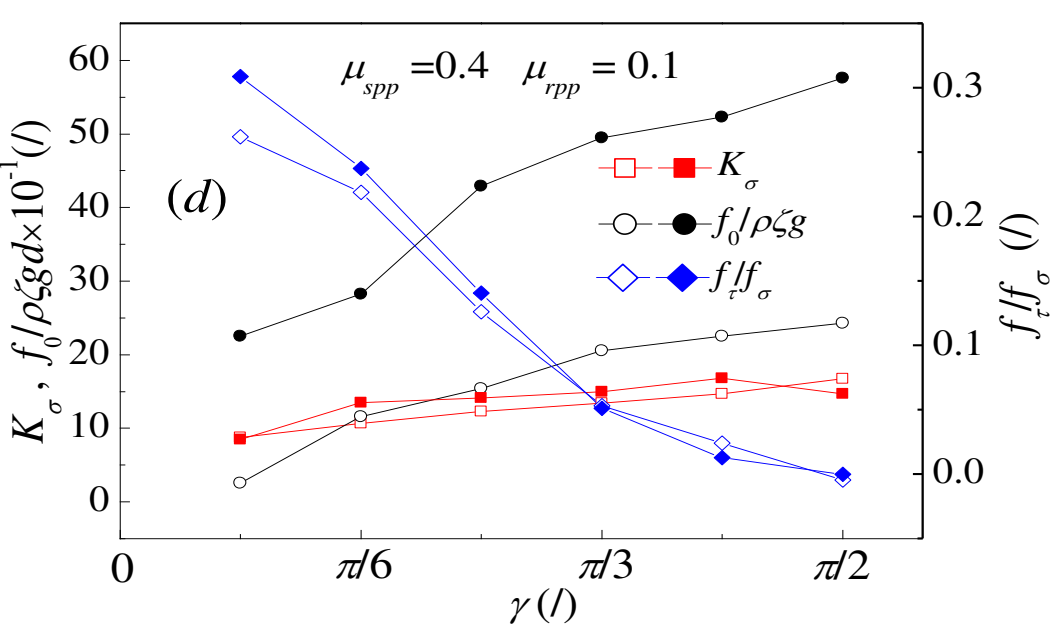

0
0
$\frac{5}{2}$
4
4
0
0
0
0
0 


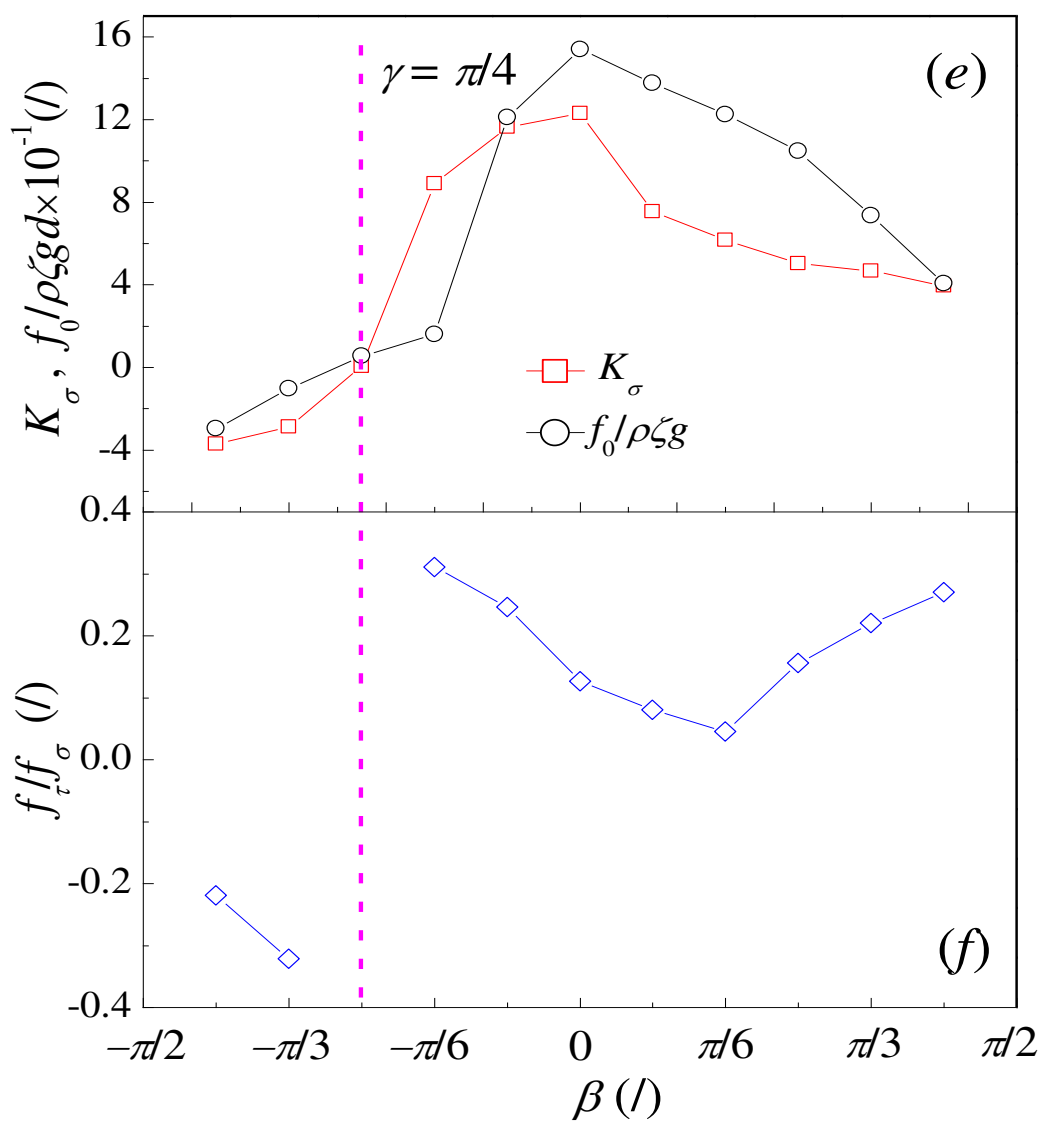




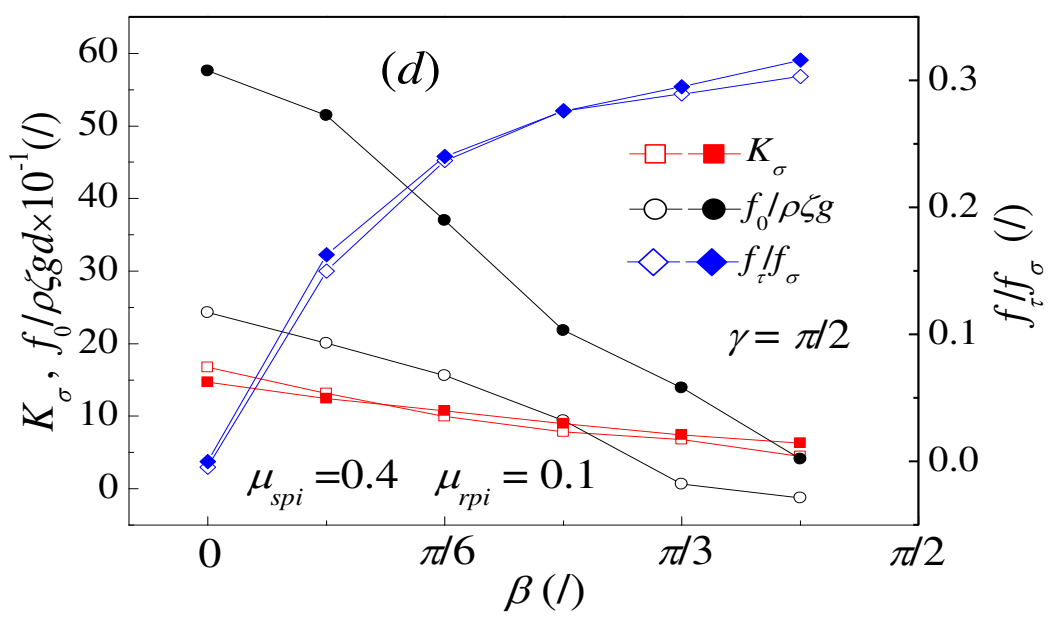

0
0
$\frac{5}{2}$
4
4
0
0
0
0
0 


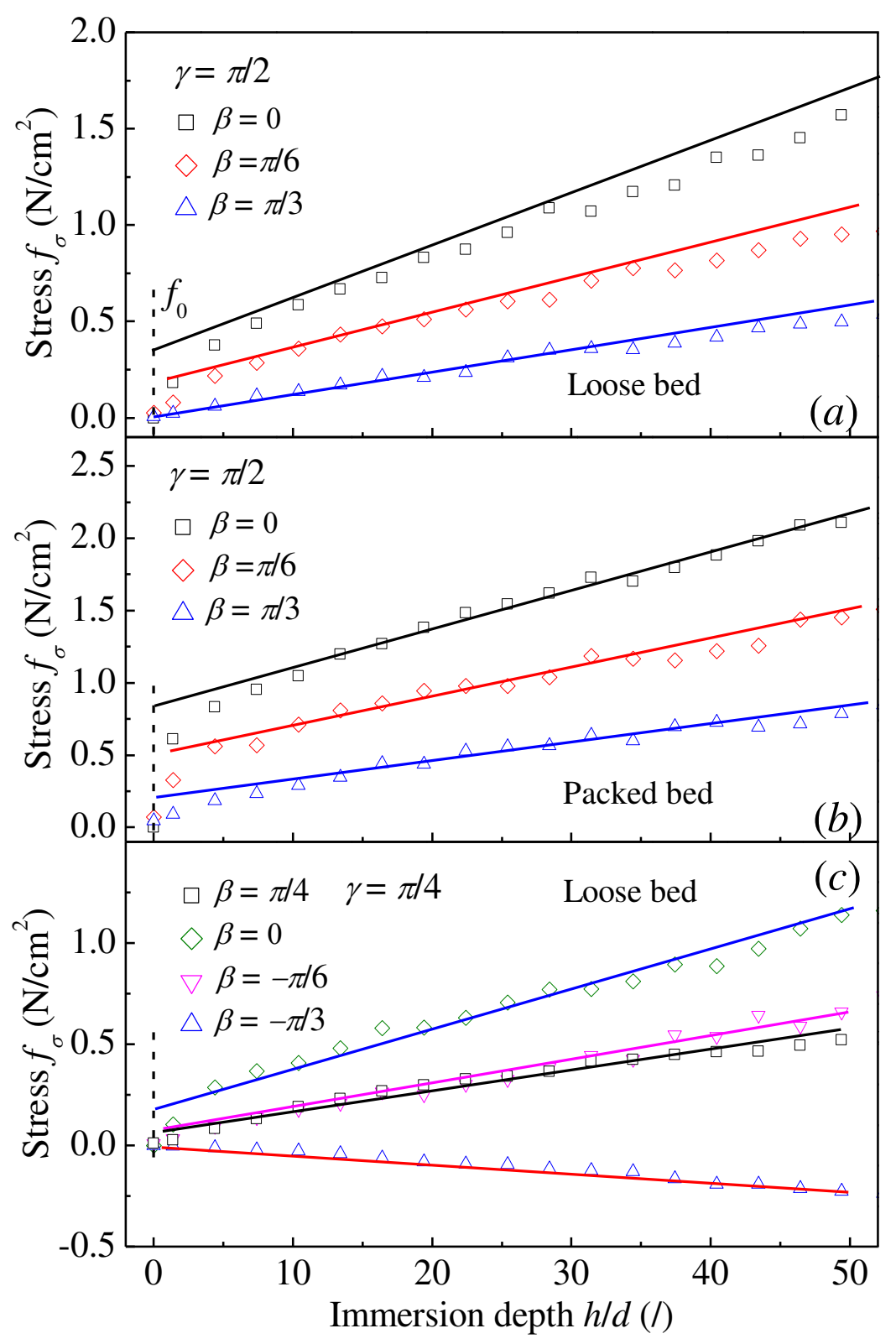




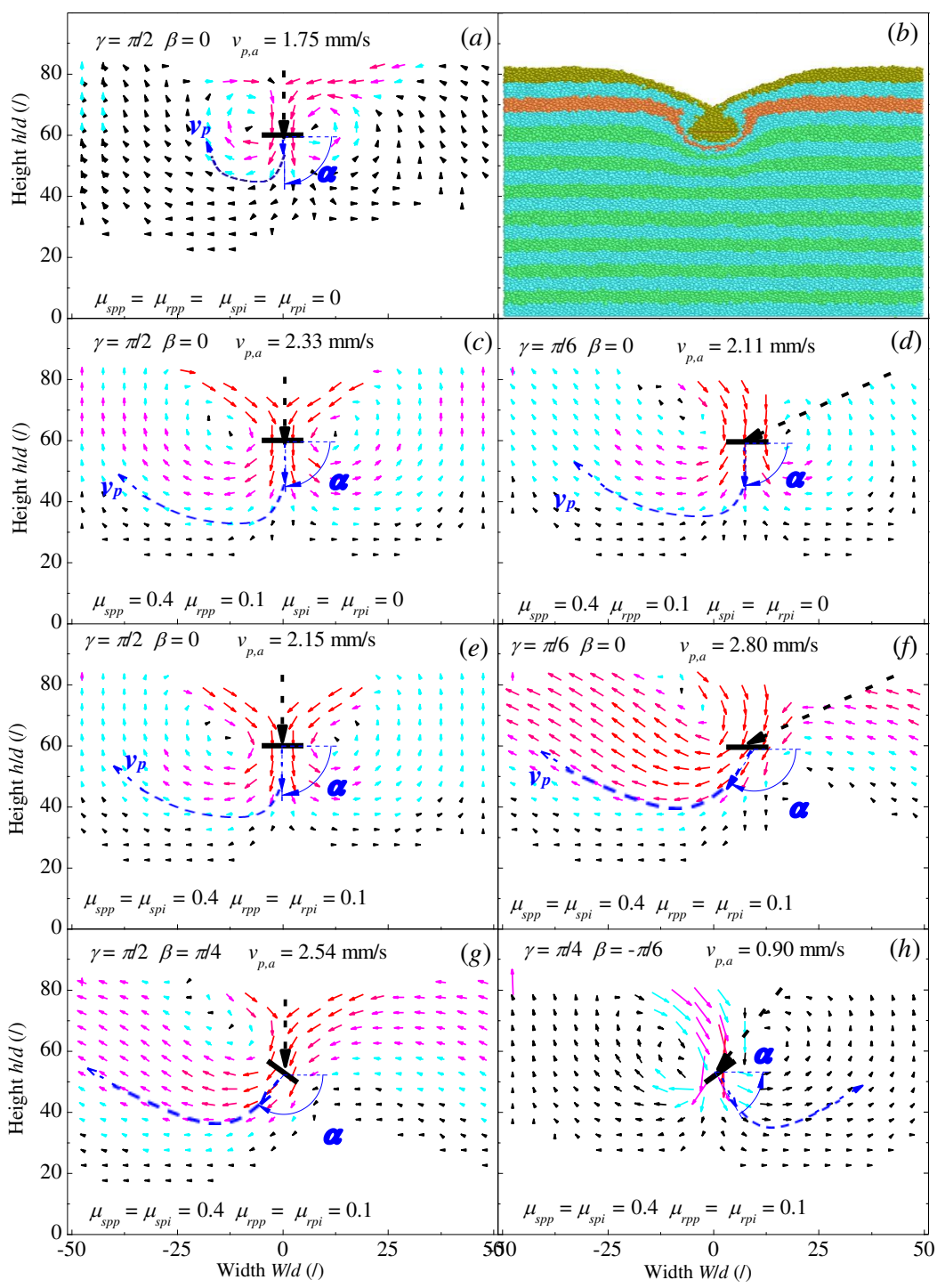




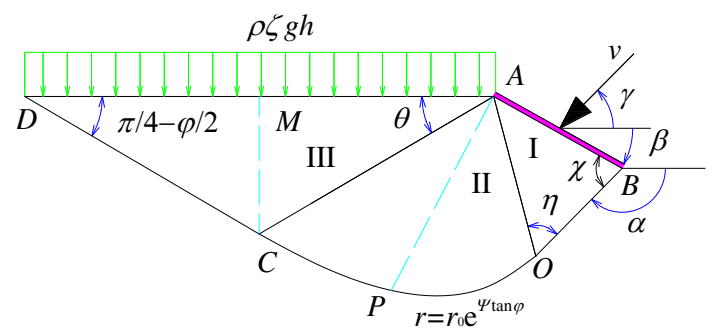

(a)

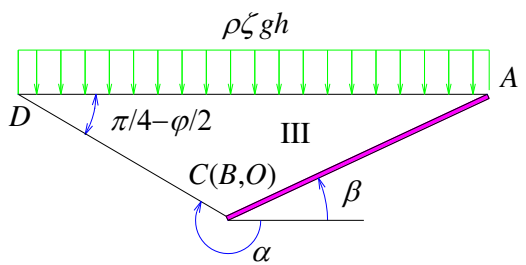

(c)

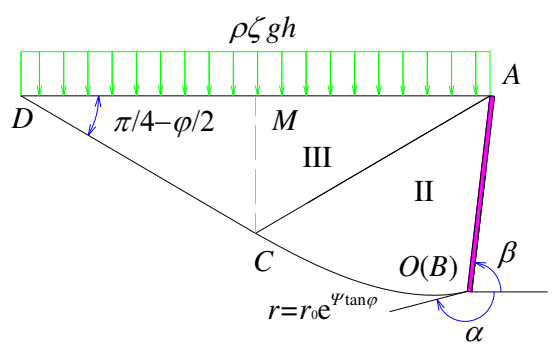

(b)

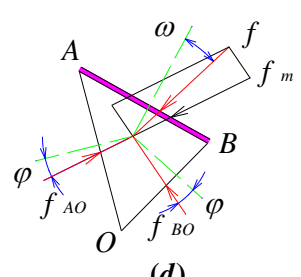




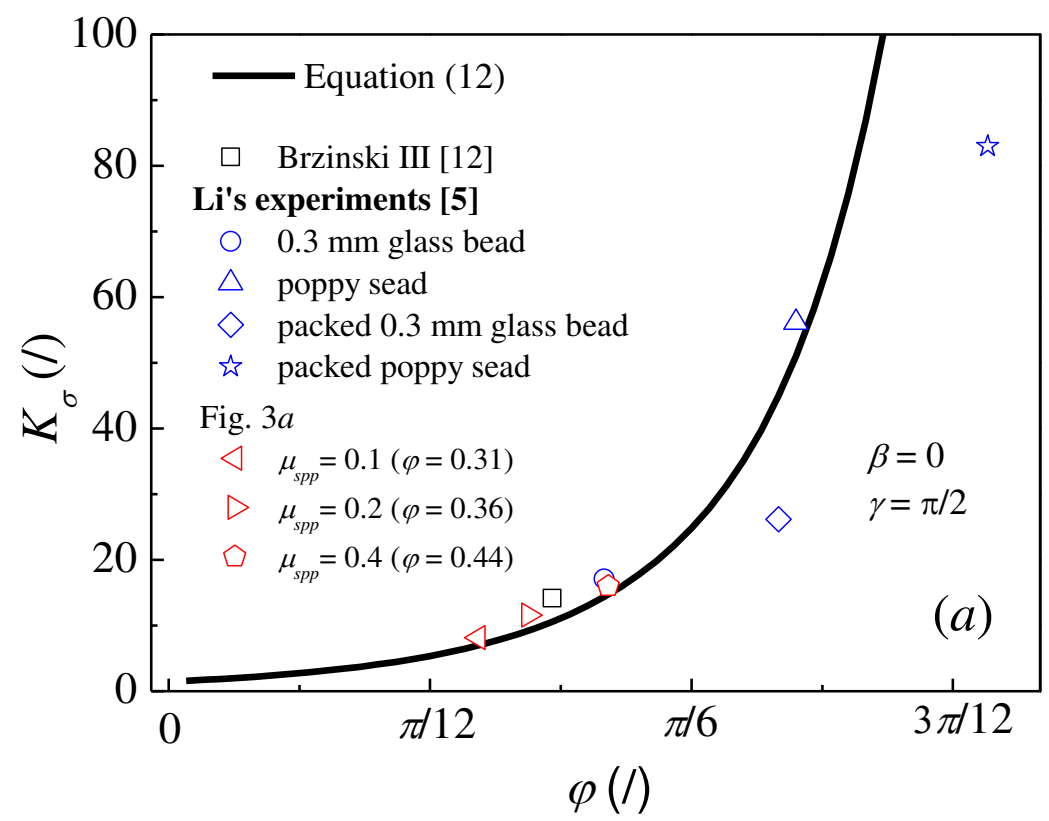




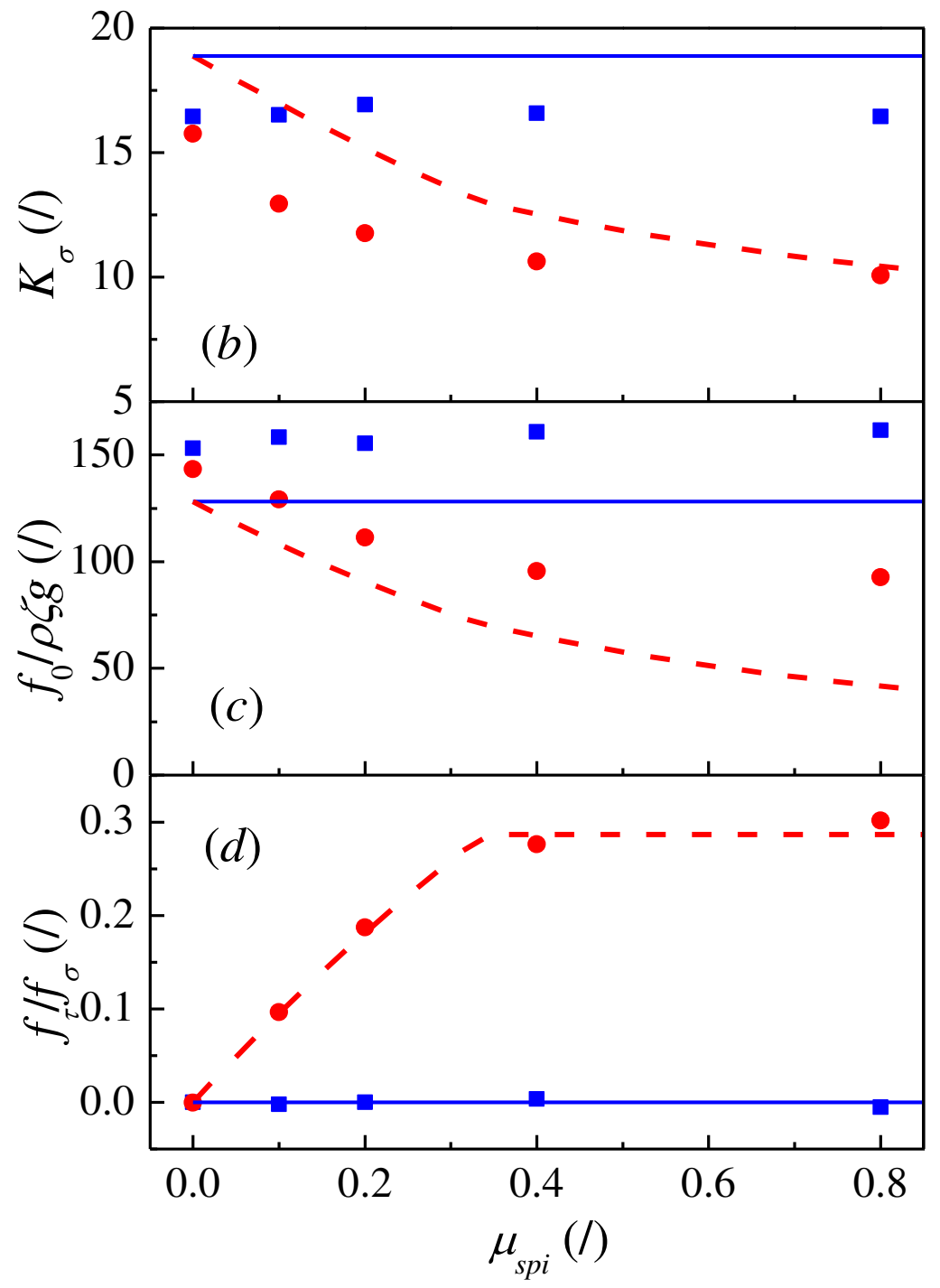

兄耪言 